\title{
RoboGraphics: Using Mobile Robots to Create Dynamic Tactile Graphics
}

\author{
Darren Guinness, Annika Muehlbradt, Daniel Szafir, Shaun K. Kane \\ University of Colorado Boulder \\ Boulder CO, USA \\ \{darren.guinness, annika.muehlbradt, daniel.szafir, shaun.kane\}@colorado.edu
}

\begin{abstract}
Tactile graphics are a common way to present information to people with vision impairments. Tactile graphics can be used to explore a broad range of content including presenting data, telling stories, and conveying map information, but aren't well suited to representing dynamic or moving information. We introduce and demonstrate a new approach RoboGraphics for creating dynamic tactile graphics by combining static tactile overlays, touch screen tablets, and off-the-shelf tangible robots. To evaluate the RoboGraphics approach for conveying information to blind users, we created a set of reference applications, including an interface for exploring graph data, braille characters, the story of the tortoise and the hare, the analog clock, and the ruminant digestive process. A design probe demonstrated that haptic graphics can be used to help people with vision impairments explore data using the audio-tactile display.
\end{abstract}

\section{Author Keywords}

Accessibility; Tangible User Interfaces; Education; Tactile; Haptic; Robots; Blindness.

\section{ACM Classification Keywords}

K.4.2. [Computers and society]: Social issues - assistive technologies for persons with disabilities.

\section{INTRODUCTION}

Recently there has been a large focus placed on accessible online media such as images, and videos. Given the opportunity to engage a larger audience, online platforms such as Twitter have made efforts to make their content accessible to people with vision impairments. Twitter has enabled the sharing of accessible images via user-uploaded captions [3]. Facebook uses a trained captioning system which can automatically provide short descriptions for useruploaded images [11]. For making videos and other recorded content accessible, Netflix [1] and YouDescribe [12] have focused on adding audio descriptions content which adds an audio channel describing the events in the video. However,

Permission to make digital or hard copies of part or all of this work for personal or classroom use is granted without fee provided that copies are not made or distributed for profit or commercial advantage and that copies bear this notice and the full citation on the first page. Copyrights for thirdparty components of this work must be honored. For all other uses, contact the Owner/Author.

ASSETS '19, October 28-30, 2019, Pittsburgh, PA, USA

(C) 2019 Copyright is held by the owner/author(s).

ACM ISBN 978-1-4503-6676-2/19/10.

https://doi.org/10.1145/3308561.3354597

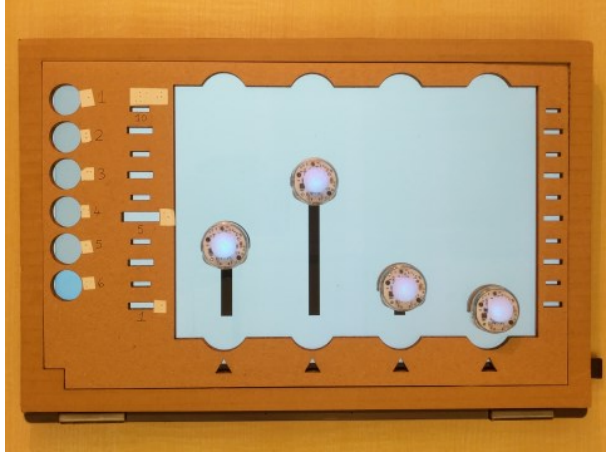

Figure 1. In the tactile bar chart app, robots form a tangible version of the bar chart on the display.

these types of text and audio descriptions typically must be concise, which can limit the ability to capture what's happening in the scene such as following how each person moves through a choreographed dance, or how the group's positioning forms distinct shapes.

Tactile Graphics and raised line drawings have recently been used to can help convey images and diagrams through touch $[9,10]$ such as those that the dancers are displaying. However, these static graphics have limited to no interactivity or support for probing different parts of the scene such examining a family member's choreographed motions in the performance.

Shape-changing and other robotic displays offer a new method of accessibly conveying animated content to people with vision impairments. These types of displays can be used to make virtual data tangible and show transformations between data [2,6], but are often prohibitively expensive or are not available to consumers [5]. In this work, we demonstrate an approach for creating touch-accessible graphics and animations using a mix of low-cost off-theshelf components available to the consumer, and static tactile overlays. Using this new approach, we can enable a person with vision impairments to understand and interpret diagrams and animations.

\section{ROBOGRAPHICS}

RoboGraphics consist of a hardware-software prototype comprising off-the-shelf components (a touchscreen tablet, and a set of mobile robots), a custom software layer, and a set of static tactile overlays which are placed on the display. 


\section{Display}

The current prototype uses a Lenovo Yoga 720 convertible laptop/tablet (15.6-inch, 16:9 touch screen, Windows 10) with a cardboard border to hold the tactile overlays.

\section{Static Tactile Overlays}

RoboGraphics include a passive tactile overlay made from cardboard which is designed to guide the user through the application and provide tactile anchors which can help the user track what is happening in the application. Tactile features on the overlays include circular reliefs to assist with identifying buttons, triangular reliefs used as a reference for lining up robots, and half circular robot bays used to help the user locate the "home" of the robots. Other applications have unique tactile features which convey concepts like the y-axis in graph applications, numbers on a clock, landmarks in short stories, and animal silhouettes.

\section{Robots}

Haptic feedback is provided by a set of Ozobot Bit 2.0 robots. These robots are $\sim 1$ cubic inch in volume and feature two wheels and an optical sensor for guidance. We chose these robots because of their versatility, ergonomic size, and low cost (about \$60 USD). As explored in [5], mobile robots such as these can be used as moving tactile "pixels" as they can be touched while driving across a surface. Ozobots are controlled using a series of color codes beneath the robot, enabling them to be dynamically controlled by a tablet.

\section{APPLICATIONS}

To examine how RoboGraphics might be used in different application contexts we conducted a design probe with a set of reference applications. The reference applications were designed to help participants understand the concept of moving tactile graphics, which are relatively new to people with vision impairments $[5,7,8]$, and to provide an initial springboard for soliciting new design ideas from participants. Details of our design process and the resulting application may be found in our paper [4].

\section{Physical Encodings and Formats}

RoboGraphics can be constructed to teach spatial encodings, structures, and concepts. For example, in the Braille Assistant a user can use the buttons to select a character to be displayed. Once selected, the system first announces the character using a speech synthesizer, and the user can discover the character by touch.

In the Analog Clock app, a user can enter a time to be presented via spoken commands or using the keyboard. The system then presents each clock hand's position at the current time using the mobile robots. The clock hands can be identified using the unique tactile mounts on top the robots.

\section{Short Stories and Interactive Explorations}

RoboGraphics can be used as a modality to explore stories and other linear content. The Tortoise and the Hare Haptic Graphic allows a user to explore a short rendition of the iconic race by touch using mobile robots featuring mounted tactile silhouettes of their character to act out the short story.

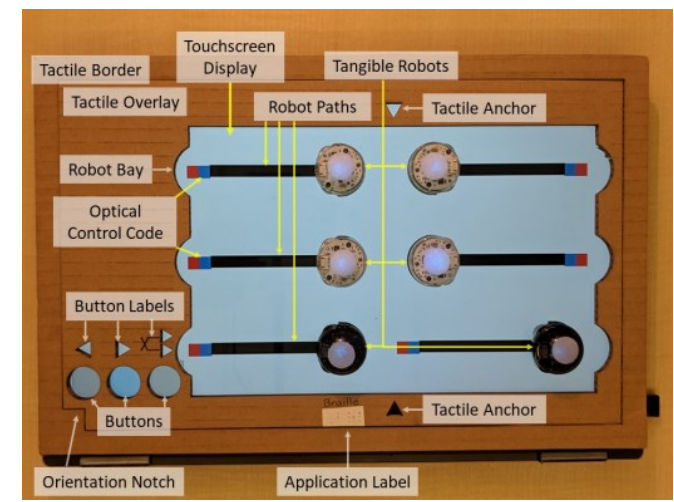

Figure 2. In the Braille Assistant Haptic Graphic, robots form a large version of the letter " $Q$ " (:) in Braille.

The graphic is accompanied by an audio narration of the story allowing the user to listen to the story and feel the events happening in the race. The user can also control the playback of the story using the buttons on the tactile overlay.

In the digestion system app, a user can follow the process of ruminant digestion in a cow to better understand how ruminants eat and regurgitate their food for further digestion. The app offers an interactive exploration providing the location of the organ via the moving robot, a sonification of the food at each organ, and a description of how the organ functions in the digestive process.

\section{Tangible Visualizations}

RoboGraphics can also be extended to support tangible data explorations similar to [9]. In the tactile bar chart app, a person with vision impairments can examine several small charts quickly using the robot's locations as tangible anchors to the data point's value. When a graph is first displayed the system speaks out a short description of the graph including the title of the graph displayed, the minimum and maximum values of the $y$-axis, and the number of units in between each of the y-axis labels on the cardboard overlay. This allows a user to quickly understand the relative differences between each of the tangible data points, but also allows the user to identify absolute differences data points or a single data point's value.

\section{CONCLUSION}

In this paper, we introduce the RoboGraphics system, a lowcost hardware-software prototype for creating dynamic tactile graphics. Our display uses a mix of off-the-shelf components and cut cardboard to enable a blind or visually impaired user to examine accessible data graphics, stories, and interactive applications.

\section{ACKNOWLEDGMENTS}

This work was supported by the National Science Foundation under grants IIS-1619384 and IIS-1652907. Any opinions, findings, conclusions or recommendations expressed in this work are those of the authors and do not necessarily reflect those of the National Science Foundation. 


\section{REFERENCES}

[1] American Council of the Blind - Netflix Audio Described Titles http://acb.org/adp/netflixad.html

[2] Sean Follmer, Daniel Leithinger, Alex Olwal, Akimitsu Hogge, and Hiroshi Ishii. 2013. inFORM: dynamic physical affordances and constraints through shape and object actuation. In Proceedings of the 26th annual ACM symposium on User interface software and technology (UIST '13). ACM, New York, NY, USA, 417-426. DOI: https://doi.org/10.1145/2501988.2502032

[3] Cole Gleason, Patrick Carrington, Cameron Cassidy, Meredith Ringel Morris, Kris M. Kitani, and Jeffrey P. Bigham. 2019. "It's almost like they're trying to hide it”: How User-Provided Image Descriptions Have Failed to Make Twitter Accessible. In The World Wide Web Conference (WWW '19), Ling Liu and Ryen White (Eds.). ACM, New York, NY, USA, 549-559. DOI: https://doi.org/10.1145/3308558.3313605

[4] Darren Guinness, Annika Muehlbradt, Daniel Szafir, and Shaun K. Kane. 2019. RoboGraphics: Dynamic Tactile Graphics Powered by Mobile Robots. In Proceedings of the 21st International ACM SIGACCESS Conference on Computers and Accessibility (ASSETS '19). ACM, New York, NY, USA. DOI: https://doi.org/10.1145/3308561.3353804

[5] Darren Guinness, Annika Muehlbradt, Daniel Szafir, and Shaun K. Kane. 2018. The Haptic Video Player: Using Mobile Robots to Create Tangible Video Annotations. In Proceedings of the 2018 ACM International Conference on Interactive Surfaces and Spaces (ISS '18). ACM, New York, NY, USA, 203211. DOI: https://doi.org/10.1145/3279778.3279805

[6] How tech helped blind students 'watch' the solar eclipse, 2017. https://www.cnet.com/news/how-techhelped-blind-students-watch-the-solar-eclipse/
[7] Annika Muehlbradt, Madhur Atreya, Darren Guinness, and Shaun K. Kane. 2018. Exploring the Design of Audio-Kinetic Graphics for Education. In Proceedings of the 20th ACM International Conference on Multimodal Interaction (ICMI '18). ACM, New York, NY, USA, 455-463. DOI:

https://doi.org/10.1145/3242969.3243004

[8] Ryo Suzuki, Abigale Stangl, Mark D. Gross, and Tom Yeh. 2017. FluxMarker: Enhancing Tactile Graphics with Dynamic Tactile Markers. In Proceedings of the 19th International ACM SIGACCESS Conference on Computers and Accessibility (ASSETS '17). ACM, New York, NY, USA, 190-199. DOI: https://doi.org/10.1145/3132525.3132548

[9] Shaun K. Kane and Jeffrey P. Bigham. 2014. Tracking (a)stemxcomet: teaching programming to blind students via $3 \mathrm{D}$ printing, crisis management, and twitter. In Proceedings of the 45th ACM technical symposium on Computer science education (SIGCSE '14). ACM, New York, NY, USA, 247-252. DOI: https://doi.org/10.1145/2538862.2538975

[10] Abigale Stangl, Jeeeun Kim, and Tom Yeh. 2014. 3D printed tactile picture books for children with visual impairments: a design probe. In Proceedings of the 2014 conference on Interaction design and children (IDC '14). ACM, New York, NY, USA, 321-324. DOI: https://doi.org/10.1145/2593968.2610482

[11] Shaomei Wu, Jeffrey Wieland, Omid Farivar, and Julie Schiller. 2017. Automatic Alt-text: Computergenerated Image Descriptions for Blind Users on a Social Network Service. In Proceedings of the 2017 ACM Conference on Computer Supported Cooperative Work and Social Computing (CSCW '17). ACM, New York, NY, USA, 1180-1192. DOI: https://doi.org/10.1145/2998181.2998364

[12] YouDescribe - Audio Descriptions for YouTube videos, 2019. https://youdescribe.org 